\title{
Article \\ A Two-Stage Scheduling Model for the Tunnel Collapse under Construction: Rescue and Reconstruction
}

\author{
Hongjun Cui ${ }^{1}$, Lijun Liu ${ }^{1}$, Ying Yang ${ }^{1}$ and Minqing $\mathrm{Zhu}{ }^{2, *}$ \\ 1 School of Civil and Transportation Engineering, Hebei University of Technology, Tianjin 300401, China; \\ cuihongjun@hebut.edu.cn (H.C.); 201831604027@stu.hebut.edu.cn (L.L.); \\ 201811601001@stu.hebut.edu.cn (Y.Y.) \\ 2 School of Architecture and Art Design, Hebei University of Technology, Tianjin 300401, China \\ * Correspondence: 2018005@hebut.edu.cn
}

Citation: Cui, H.; Liu, L.; Yang, Y.; Zhu, M. A Two-Stage Scheduling

Model for the Tunnel Collapse under Construction: Rescue and

Reconstruction. Energies 2022, 15, 743 https://doi.org/10.3390/en15030743

Academic Editor: Balázs Németh

Received: 12 November 2021

Accepted: 11 January 2022

Published: 20 January 2022

Publisher's Note: MDPI stays neutral with regard to jurisdictional claims in published maps and institutional affiliations.

Copyright: (C) 2022 by the authors. Licensee MDPI, Basel, Switzerland. This article is an open access article distributed under the terms and conditions of the Creative Commons Attribution (CC BY) license (https:// creativecommons.org/licenses/by/ $4.0 /)$.

\begin{abstract}
In the process of transportation system construction, the tunnel is always an indispensable part of the traffic network due to terrain constraints. A collapse of the tunnel under construction may give rise to a potential for significant damage to the traffic network, complicating the road conditions and straining relief services for construction workers. To cope with the variety of vehicle types during the rescue effort, this paper divides them into small, medium, and large sizes, herein correcting the corresponding speed considering six road condition factors on account of the previous research. Given the influence of different special road conditions on the speed of different sized vehicles, a multi-objective model which contains two stages is presented to make decisions for rescue vehicle scheduling. Under the priority of saving human life, the first-stage objective is minimizing the arrival time, while the objective of the second stage includes minimizing the arrival time, unmet demand level, and scheduling cost. To solve the currently proposed model, a non-dominated sorting genetic algorithm II (NSGA-II) with a real number coding method is developed. With a real tunnel example, the acceptability and improvement of the model are examined, and the algorithm's optimization performance is verified. Moreover, the efficiency of applying real number coding to NSGA-II, the multi-objective gray wolf algorithm (MOGWO), and the traditional genetic algorithm (GA) is compared. The result shows that compared with the other two methods, the NSGA-II algorithm converges faster.
\end{abstract}

Keywords: tunnel collapse; emergency rescue; vehicle scheduling; multi-vehicle size; NSGA-II

\section{Introduction \\ 1.1. Background}

For mountainous countries such as China, the tunnel is an essential component of the transportation network. However, with the increase of road construction mileage, the rate of tunnel collapse accidents under construction also rises. According to the characteristics of high frequency and risk of tunnel collapse under construction, the accidents threaten the safety of constructers, causing casualties and economic loss. There were 49 tunnel collapse accidents under construction from 2006 to 2018 in China, with 255 casualties. The severe collapse of the accidents is listed in Table 1.

Proper operations to reduce human suffering and death are necessary. In the previous research, to cope with the sudden tunnel collapse under construction, the emergency management operations consist of monitoring and warning, emergency rescue response, and reconstruction in post-disaster. Among them, the emergency rescue response, which includes the supply scheduling and on-site rescue activities, directly affects the safety of the constructors and the economic loss. How to provide help to the affected area as soon as possible is of great importance for it is the vital prerequisite of the on-site rescue activities. One mechanism to avoid unnecessary time delays is the effective utilization and sufficient 
provision of a rescue service with strategic scheduling plans, which allows for mitigating damage and loss in disasters.

Table 1. Collapse accidents of tunnels under construction.

\begin{tabular}{|c|c|c|c|c|}
\hline \multirow{2}{*}{ Date } & \multirow{2}{*}{ Tunnel } & \multicolumn{2}{|c|}{ Casualty } & \multirow{2}{*}{$\begin{array}{l}\text { Economic Loss } \\
\text { (Thousand) }\end{array}$} \\
\hline & & Trapped & Death & \\
\hline 3 May 2014 & $\begin{array}{c}\text { Anhui } \\
\text { Longtouling }\end{array}$ & 8 & 6 & 8000 \\
\hline 5 December 2014 & Fujian Housi & 21 & - & 6500 \\
\hline 20 August 2016 & Guihzou Zhubu & 7 & 7 & 3245.6 \\
\hline 14 September 2017 & Yunnan Manyao & 9 & - & 1255.8 \\
\hline 30 September 2017 & Hebei Yingerling & 9 & - & 629.8 \\
\hline
\end{tabular}

However, the tunnels under construction are mainly located on sections with complicated surrounding conditions, with possible unopened sections on the routes for supplies' transportation. The complex road environment brings challenges to scheduling and transporting rescue supplies. What is worse, the emergency rescue vehicles (includes special vehicles such as ambulances, engineering rescue vehicles, cranes, etc.) differentiate according to the characteristics of ultra-high, ultra-wide, and heavy-duty, requiring special road conditions for passing. This highlights the need for suitable vehicle scheduling routes with characteristics that could minimize the limitation of passage for different sizes of vehicles, therefore ensuring the arrival time of rescue efforts, saving the lives of trapped constructors, and reducing property losses. Obviously, the improvement of decisions made considering the road conditions and vehicle sizes could result in the high efficiency of rescue operations.

Specializing in making optimal decisions of the vehicle scheduling, this paper proposes a two-stage multi-objective vehicle scheduling model that includes three vehicle sizes to tackle the limitations of special conditions that influence the passage of rescue vehicles. Firstly, regarding the quantified influence of the road conditions on vehicle speed, the correction coefficients based on the previous research [1] are presented to help answer this question. Secondly, we propose a two-stage model to determine the scheduling plan of rescue vehicles according to their size to reduce the impact of road conditions. Thus, the emergency service could be provided as soon as possible, which means a lot to the trapped survivors and the rescue and reconstruction work. At the same time, the design of the two stages in this model specifies the priority in the different stages of operations. Human lives will be saved first, which is the main idea of emergency management. Then, the economic loss and the reconstruction work are taken into account. Thirdly, we constructed an NSGA-II solution algorithm to solve the proposed model. In the previous modeling approach [2,3], a binary variable of multiple indices that define the vehicle and route identification and starting and ending nodes is applied to denote the vehicle route. When the number of routes and vehicles used in operation is large, the number of binary variables increases significantly, leading to a model of restricted applicability. In this research, a real number coding method is applied to reduce the complexity of the solution process. Finally, a comparison of the NSGA-II algorithm, the multi-objective gray wolf algorithm (MOGWO), and the general genetic algorithm (GA) are provided.

\subsection{Literature Review}

Humanitarian logistic alias vehicle scheduling has been an active area of research in the recent past to reduce human life and economic loss by both natural and man-made disasters. To enhance the efficiency of emergency management, humanitarian logistics, also called emergency supply, which focuses on providing rescue supplies (emergency shelter, food, water, or medicine) to the area in demand timely, is critical. As a part of humanitarian logistics, the Vehicle Scheduling Problem (VSP) can be formulated as an optimization problem that outputs an optimal plan. This plan determines which vehicle to 
employ to transport the supplies from one (or multiple) rescue point(s) to the affected area and the number of vehicles scheduled.

Substantively, the Vehicle Scheduling Problem (VSP) includes serious content, such as the Distribution Problem, the Vehicle Routing Problem (VRP), etc. Given the literature consulted at present, the least attention has been paid to the scheduling plan of different sized rescue vehicles studied in this paper, especially in the collapse disasters of a tunnel under construction compared with other disaster management operations. Thus, the related research on improving the operations of the distribution, routing design, and other rescue activities against the disasters is surveyed and reviewed in this section.

The two-stage stochastic (2SS) is the main component in the rescue operations of emergency management [4]. Generally, the first-stage decisions are made without knowing the random parameters. According to the results acquired, the second-stage recourse variables are decided [5]. Decisions of the scheduling problem consist of the supply points that provide help, the number of supplies transported by each vehicle, the arrangement of the rescuers, the routing, etc. In the first stage, the decisions are primarily the scheduling of the vehicles that provide aid assistance. While in the second stage, the main idea of decisions is reconstruction. For detailed information on the application of the two-stage model, see [6-10]. To cope with the uncertainty of the disaster, stochastic approaches are implemented [9]. In the early studies, research is mainly about one rescue point to one demand point. Later, the work was extended from one rescue point to multiple demand points and multiple rescue points to multiple demand points.

Although the distinctions between commercial logistics and humanitarian relief chains have been explicated by scholars [11-14], cost-minimization (purchase, storage, transportation, etc.) is still a common trend in both pre- and post-disaster planning problems in the emergency operations $[5,7,12,15-19]$. Additionally, the objectives also cover the investment budget, the total travel time and the distance [20-22], the safety, the unmet demand evaluated by indicators such as the demand satisfaction rate [23], and equity among demand points. [24,25]. Focusing on pre-positioning the relief items, Paul and MacDonald [19] proposed a model intending to minimize the total cost of expected fatalities. In the research of Elçi and Noyan [18], the optimal objective is to minimize all the operating costs.

As the decision-makers tend to achieve multiple objectives, scholars integrated the multi-objective planning problem into the emergency management operations. Research on the multi-objective model in the design of emergency logistics has also been extensively reported. Mohamadi and Yaghoubi [21] developed a bi-objective stochastic model to minimize transportation time and costs. Bozorgi-Amiri and Khorsi [7] proposed a model consisting of three objective functions: minimizing the costs of shortages, transportation time, and all the expected operations. When solving the proposed model, the solution method consists of the heuristic algorithm [26], particle swarm optimization [27], the ant colony algorithm [28], etc. In addition, the hierarchical solution is used to optimize the objective of the first stage, and the optimal solution obtained is added as a constraint to the model to make the second optimization goal optimal. For the multi-objective approaches, Mohammadi et al. [29] obtained a Pareto front, while Dalal and Üster [30] applied the $\varepsilon$-constraint method. The weighted sum of objectives is considered as well.

Based on the above discussion, some gaps have not been considered in detail. Firstly, as explained before, the tunnels under construction are mostly located on remote sections with complex terrain, of which the condition affects the driving of various rescue vehicles. In addition, rescue vehicles of the tunnel collapse accidents consist of ambulances and large-sized special vehicles, such as engineering rescue vehicles. Therefore, the influence of road conditions and vehicle size needs further consideration. Secondly, according to abrupt and devastating effects, both trapped people and the reconstruction need to be considered in rescue operations under the underlying principle of saving lives first. This means objectives have different priorities in two stages (rescue and reconstruction stages). Therefore, the two-stage vehicle scheduling research is carried out in this paper according to the vehicle size and road conditions for the decision-making, as follows. 


\subsection{Contributions of This Research}

Firstly, according to the number of axes and length, the vehicles are divided into three sizes in this paper to deal with the complex composition of rescue vehicle types. Then, as to the influence of special road conditions on the vehicle speed with different sizes, the corresponding speed is corrected based on the previous research. Secondly, in combination with the priority of different objectives in the first stage (rescue stage) and the second stage (reconstruction stage), a multi-objective model is proposed for vehicle scheduling decision-making. Thirdly, an NSGA-II algorithm with a real number coding method is developed to solve the model proposed. With a real example tunnel under construction, the practicability and the improvement of the model are examined. Finally, the efficiency of the NSGA-II algorithm is verified through the comparison with the multi-objective gray wolf algorithm (MOGWO) and the general genetic algorithm (GA).

This paper is organized as follows: In Section 1, the background, the related studies, and the main work of this research have been introduced in detail. Section 2 describes the problem of interest, including the definitions and assumptions of the proposed model, as well as the speed correction process of different sized vehicles on various road conditions for acquiring the corresponding travel time. These two sections lay the groundwork for the propositions in this research. In Section 3, the proposed mathematical model together with a solution algorithm is presented. Section 4 implements the proposed model to a real case. Section 4 also covers the comparison of two coding algorithms' efficiency. Finally, the conclusion and prospects are summarized in Section 5.

\section{Problem Description}

\subsection{Definitions and Assumptions}

Let $G=(V, A)$ represent the road network of interest. $V$ is the set of nodes in the transportation network, and $A$ is the set of road sections (links) between the nodes. Suppose node $o$ in the topological network is the tunnel where the accident happened and $J$ is the set of vehicle supply nodes. It is assumed that the vehicle supply points are located at the transport nodes in the research area $o, J \in V$.

In case of a disaster, the supply nodes should provide help to the affected tunnel. In association with the complexity of rescue vehicles' composition, this paper divides them into three sizes, including small, medium, and large, which are denoted by $m$, according to the number of axles and length, as shown in Table 2.

Table 2. Rescue vehicle classification.

\begin{tabular}{cccc}
\hline Size & Example & Number of Axes & Length (m) \\
\hline Small & Ambulance, light truck, etc. & 2 & 5.5 \\
Medium & Supply transport vehicles, large fire trucks, etc. & $3-5$ & $5.5-11.5$ \\
Large & Transport cranes, truck cranes, excavator trailers, etc. & $>5$ & $>11.5$ \\
\hline
\end{tabular}

In the first stage, with the priority of saving lives, the objective is minimizing the arrival time of rescue vehicles. We used the time interval between when the accident happens and the arrival of the last rescue vehicle here to indicate this objective.

In the second stage, after finishing rescuing the survivors, the reconstruction work begins. Three objectives, minimizing the average arrival time, the unmet demand, and the scheduling cost, are considered at this time. The rescue vehicle scheduling modeling is based on the following assumptions:

(a) There is no capacity limitation at the rescue point. Only the number of rescue vehicles is limited.

(b) Emergency supplies can be mixed in one rescue vehicle according to the characteristics of the emergency supplies and the vehicle types.

(c) The laytime is not considered here. The time interval of the accident and the rescue vehicle's arrival is the travel time on the road. 
(d) The demand in the affected tunnel is known, and depends on the survey's situation at different stages of the collapse.

The definitions and notations in this paper are described in Table 3.

Table 3. Notations and definitions.

\begin{tabular}{|c|c|}
\hline Notations & Definitions \\
\hline$o$ & the affected point \\
\hline$J$ & the set of rescue points \\
\hline$j$ & the rescue point $j, j \in J$ \\
\hline$V$ & the set of transportation nodes in the network, $o, J \in V$ \\
\hline$N$ & the number of transportation nodes in the network \\
\hline$v_{i}$ & the transportation node $i$ in the traffic network, $v_{i} \in V, i=1,2,3 \ldots N$ \\
\hline$A$ & the set of road traffic sections \\
\hline$a$ & the road traffic section $a, a \in A$ \\
\hline G & the transportation network, $G=(V, \mathrm{~A})$ \\
\hline$m$ & the size of the rescue vehicles, $m \in M$ \\
\hline$u$ & the number of rescue points in the transportation network of interest \\
\hline$u_{j}$ & the number of total available vehicles in the rescue point $j$ \\
\hline$v_{a}^{d m}$ & the design speed of $m$ size vehicle on the road section $a$ \\
\hline$v_{a}^{r m}$ & the real speed of $m$ size vehicle on the road section $a$ \\
\hline$l_{a}$ & the distance of the road section $a$ \\
\hline$t_{a}^{m}$ & the travel time of $m$ size vehicle on the road section $a$ \\
\hline$L_{j o}$ & the distance between the rescue point $j$ and the accident point $o$ \\
\hline$\tau_{m_{j}}$ & the shortest travel time of $m$ size vehicle from the rescue node $j$ to the affected node $o$ \\
\hline$T$ & the vehicle arrival restriction period \\
\hline$x_{m_{j}}^{f}$ & the number of $m$ size vehicles distributed from the rescue node $j$ in the first stage \\
\hline$x_{m_{j}}^{\mathrm{s}}$ & the number of $m$ size vehicles distributed from the rescue node $j$ in the second stage \\
\hline$w_{m_{j}}$ & the original number of $m$ size vehicles available at therescue point $j$ \\
\hline$d_{m}^{f^{\prime}}$ & the number of $m$ size vehicles in demand of the affected node $o$ in the first stage \\
\hline $\begin{array}{l}d_{m}^{s} \\
b\end{array}$ & $\begin{array}{l}\text { the number of } m \text { size vehicles in demand of the affected node } o \text { in the second stage } \\
\text { the total amount of supplies required for rescue }\end{array}$ \\
\hline$q^{m}$ & the maximum loading capacity of $m$ size vehicle \\
\hline$k_{i}$ & the speed correction coefficient $i, k_{i} \in K, i=1,2,3 \ldots 6$ \\
\hline$\lambda$ & the horizontal curve radius \\
\hline$\theta$ & the gradient \\
\hline$L_{S}$ & the length of the longitudinal slope \\
\hline$\alpha$ & the actual traffic volume \\
\hline$\beta$ & the proportion of large vehicles in the section \\
\hline$\partial$ & the punishment coefficient for the delay of the rescue supplies \\
\hline$P C I$ & the pavement condition index \\
\hline$c_{t}$ & the unit distance travel cost of the $m$ size vehicle \\
\hline$z_{i}$ & the unit distance travel cost of the $i, i=1,2,3$ \\
\hline$\omega_{i}$ & the weight coefficient of the corresponding optimal objective $i, i=1,2,3$ \\
\hline$r_{\max }$ & the maximum number of iterations \\
\hline$r$ & the algebra of the population generated \\
\hline$P_{r}$ & the population of generation $r$ \\
\hline$Q_{r}$ & the subpopulations of the t-generation population \\
\hline$n$ & the size of the population generated \\
\hline$p_{i}^{b s}$ & the probability of the individual $i$ being selected \\
\hline$f_{i}$ & the fitness value of individual $i$ \\
\hline$p^{c r}$ & the crossover probability \\
\hline$p^{m u}$ & the mutation probability \\
\hline
\end{tabular}

\subsection{Travel Time}

The influence of complex road conditions on vehicle driving is inevitable, for which speed correction is necessary according to the road environment and the vehicle sizes. Based on the road design speed, this paper corrects the vehicle speed with the correction 
coefficients, including horizontal curve radius $k_{1}$, longitudinal slope $k_{2}$, traffic flow $k_{3}$, traffic composition $k_{4}$, road surface $k_{5}$, and special factors $k_{6}$.

The actual vehicle speed is defined as follows:

$$
v_{a}^{r m}=\frac{k_{1} \cdot k_{2} \cdot k_{3} \cdot k_{4} \cdot k_{5}}{k_{6}} \cdot v_{a}^{d m}
$$

where the correction coefficients $k_{1}-k_{5}$ are the ratio of the actual vehicle speed to the road design speed, $v_{a}^{r m}$ is the actual speed of $m$ size vehicles on the road section $a$, and $v_{a}^{d m}$ is the design speed of $m$ size vehicle on the road section $a$.

Under the ideal state, the respective speeds of small, medium, and large type vehicles are 135,135 , and $95 \mathrm{~km} / \mathrm{h}$, respectively. Based on previous research [1], the correction coefficients of rescue vehicle speed are defined as follows:

(1) The horizontal curve radius correction coefficient:

$$
\begin{gathered}
k_{1}^{s m}=k_{1}^{m e}=0.26 \lambda^{0.1623} \\
k_{1}^{l a}=0.04 \lambda^{0.3615}
\end{gathered}
$$

where $\lambda$ indicates the horizontal curve radius, and $k_{1}^{s m}, k_{1}^{m e}$, and $k_{1}^{l a}$ are the correction coefficients of the horizontal curve radius of small, medium, and large vehicles, respectively.

(2) The longitudinal slope correction coefficient

The impact of longitudinal slope on the vehicle driving includes the longitudinal gradient and grade length, as shown in the following:

$$
\begin{gathered}
k_{2}^{s m}=\left\{\begin{array}{c}
-0.29 \theta^{2}+1.07 \theta-3.6 \times 10^{-4} L_{s} \\
+0.694, \theta \leq 3 \% \\
-0.06 \theta-3.6 \times 10^{-4} L_{s} \\
+1.53, \theta>3 \%
\end{array}\right. \\
k_{2}^{m e}=-0.12 \theta+6.9 \times 10^{-4} L_{s}+0.29,2 \% \leq \theta \leq 5 \% \\
k_{2}^{l a}=\left\{\begin{array}{c}
-0.11 \theta^{2}+0.6 \theta-2.8 \times 10^{-4} L_{s} \\
-0.12, \theta \leq 3 \% \\
-0.11 \theta-2.8 \times 10^{-4} L_{s} \\
+0.24, \theta>3 \%
\end{array}\right.
\end{gathered}
$$

where $\theta$ is the gradient, $L_{s}$ is the slope length, and $k_{2}^{s m}, k_{2}^{m e}$, and $k_{2}^{l a}$ represent the longitudinal slope correction coefficient of small, medium, and large vehicles, respectively.

(3) The traffic flow correction coefficient

The traffic flow correction coefficient is used to describe the impact of traffic flow on rescue vehicles:

$$
k_{3}=2.89 \alpha^{-0.2392}
$$

where $\alpha$ is the actual traffic volume $/\left(\mathrm{pcu} \cdot \mathrm{h}^{-1} \cdot\right.$ lane $\left.^{-1}\right)$.

(4) The traffic composition correction coefficient

Traffic composition refers to the proportion of large vehicles on straight road sections:

$$
k_{4}=8.46 \times 10^{-5} \beta_{2}-0.02 \beta+1.47
$$

where $\beta$ represents the proportion of large vehicles in the section studied.

(5) The pavement condition correction coefficient 
The pavement condition index, $P C I$, is used to indicate the road condition [31]. For the highway with a design speed of $120 \mathrm{~km} / \mathrm{h}$, the correction coefficients corresponding to different road conditions are shown in Table 4.

(6) The special factor influence coefficient

Table 4. Correction coefficients of $120 \mathrm{~km} / \mathrm{h}$ highway in different pavement conditions.

\begin{tabular}{cccc}
\hline PCI & $\begin{array}{c}\text { Correction } \\
\text { Coefficient }\end{array}$ & PCI & $\begin{array}{c}\text { Correction } \\
\text { Coefficient }\end{array}$ \\
\hline$>60$ & 1 & {$[37,39]$} & 0.76 \\
{$[54,60]$} & 0.96 & {$[35,37]$} & 0.72 \\
{$[49,54]$} & 0.92 & {$[33.7,35]$} & 0.68 \\
{$[45,49]$} & 0.88 & {$[32.8,33.7]$} & 0.64 \\
{$[41.5,45]$} & 0.84 & {$[31.8,32.8]$} & 0.6 \\
{$[39,41.5]$} & 0.8 & {$[31,31.8]$} & 0.56 \\
\hline
\end{tabular}

In the case of the road sections with limited width and height or insufficient capacity, the special rescue vehicles with characteristics including extreme height, width, and heavy load involved in the rescue of tunnel collapse find it difficult to pass through. The special factor influence coefficient is defined as shown in Equation (9):

$$
k_{6}=\left\{\begin{array}{l}
\infty, \text { road restrictions, no vehicles can pass } \\
1, \text { otherwise }
\end{array}\right.
$$

Herein, with the value $v_{a}^{r m}$ acquired from Equation (1), the travel time is calculated as follows:

$$
t_{m}^{a}=\frac{l_{a}}{v_{a}^{r m}}
$$

where $t_{m}^{a}$ denotes the travel time of $m$ size vehicle passing the section $a$, and $l_{a}$ represents the length of the road section $a$.

The shortest travel time of $m$ size vehicle from the rescue point $j$ to the accident tunnel is shown as follows:

$$
\tau_{m_{j}}=\min \sum_{a \in A, j \in J} t_{m}^{a}
$$

It is assumed that rescue work starts from the first rescue vehicle's arrival time and ends at the last vehicle's arrival time. The vehicle scheduling time is defined as the arrival time of the last rescue vehicle.

\section{The Two-Stage Vehicle Scheduling Model}

For humanitarian purposes, saving human lives is a priority in the initial rescue effort. Reconstruction will not be carried out until the trapped survivors are rescued. Based on the priority of each objective at different stages, this paper proposes a multi-size twostage model to make rescue scheduling decisions. In the first stage, minimizing the travel time is taken as the objective, so that the survivors can be saved as soon as possible. In the second stage, the total maximum scheduling time, the unmet demand, and the cost are taken into consideration for resettling the rescued trapped persons and carrying out reconstruction work.

\subsection{Mathematical Modeling}

To provide help to the collapsed tunnel, we minimize the maximum completion time of the scheduling work in the first stage as follows:

$$
T(\phi)=\min \left(\max _{\tau_{m_{j}}}+\partial\right)
$$




$$
\begin{gathered}
x_{m_{j}}^{f} \leq w_{m_{j}} \\
\sum_{j \in J} \sum_{m \in M} x_{m_{j}}^{f} \geq d_{m}^{f} \\
\partial=\left\{\begin{array}{l}
0, \text { if } \tau_{m_{j}} \leq T \\
\partial^{\prime}, \text { other }
\end{array}\right. \\
x_{m_{j}}^{f} \text { is non - negative integer }
\end{gathered}
$$

where $T(\phi)$ is the maximum completion time of the scheduled work, $\tau_{m_{j}}$ is the shortest travel time of $m$ size vehicle from rescue node $j$ to affected node $o, \partial$ is a punishment coefficient for the delay of the rescue supplies, $x_{m_{j}}^{f}$ is the number of $m$ size vehicles dispatched from rescue node $j$ to affected node $o$ in the first stage, $w_{m_{j}}$ represents the original number of $m$ size vehicles available at rescue point $j, d_{m}^{f}$ is the number of $m$ size vehicles in demand of node $o$, and $T$ is the vehicle arrival restriction period.

The objective function Equation (12) is used to minimize the travel time of the last rescue vehicle that arrived so that the rescue efforts could arrive in the shortest time. The constraint Equation (13) represents that the number of vehicles dispatched from the rescue point $j$ should be less than originally owned, while the constraint Equation (14) ensures the number of $m$ size vehicles dispatched from all supply points is not less than the number required in the affected node $o$. The constraint Equation (15) indicates that the rescue vehicle's arrival time should be within the limitations period, otherwise the objective would be affected, and the constraint Equation (16) is the decision variable constraint.

In the second stage, we use three objectives to ensure the efficiency of the rescue and reconstruction operations. The first objective is proposed as follows:

$$
Z_{1}=\min \frac{\sum_{j \in J} \sum_{m \in M} \tau_{m_{j}} x_{m_{j}}^{s}}{\sum_{j \in J} \sum_{m \in M} x_{m_{j}}^{s}}
$$

where $x_{m_{j}}^{s}$ is the number of $m$ size vehicles dispatched from rescue node $j$ to affected node $o$ in the second stage. The objective function Equation (17) means minimizing the average arrival time of rescue vehicles to ensure the timely arrival of rescue vehicles.

At the same time, for sufficient relief supply, the objective function Equation (18) is proposed to minimize the unmet demand:

$$
\mathrm{Z}_{2}=\min \left(b-\sum_{j \in J} \sum_{m \in M} q^{m} x_{m_{j}}^{s}\right)
$$

where $b$ represents the total amount of supplies required for rescue (the volume and weight of all supplies, including cranes to be transported, etc.), and $q^{m}$ is the maximum loading capacity of $m$ size vehicles.

Besides, the objective of minimizing the cost has been widely used:

$$
Z_{3}=\min \sum_{j \in J} \sum_{m \in M} c_{t} L_{j o} x_{m_{j}}^{s}
$$

where $c_{t}$ is the unit distance travel cost of the $m$ size vehicle (fuel costs, road and bridge tolls, etc.), and $L_{j o}$ denotes the distance between the rescue point $j$ and the accident point $o$. The objective function Equation (19) represents minimizing the rescue vehicle scheduling cost.

$$
\begin{gathered}
x_{m_{j}}^{s} \leq w_{m_{j}}-x_{m_{j}}^{f} \\
\sum_{j \in J} \sum_{m \in M} x_{m_{j}}^{s} \geq d_{m}^{s}
\end{gathered}
$$




$$
\begin{gathered}
\sum_{j \in J} \sum_{m \in M} x_{m_{j}}^{s} q^{m} \geq b \\
x_{m_{j}}^{s} \text { is non-negative integer }
\end{gathered}
$$

where $d_{m}^{s}$ is the number of $m$ size vehicles in demand.

Constraint Equation (20) indicates that the number of vehicles dispatched from the rescue point $j$ should be less than the remaining available amount. Constraint Equations (21) and (22) show that the number of vehicles and supplies distributed should not be less than the required amount. Constraint Equation (23) is a non-negative constraint.

\subsection{Solution for the Model}

In the first stage, the vehicles are scheduled from the rescue points near the affected node. Therefore, when solving the model in the first stage, $\tau_{m_{j}}$ is sorted for $m$ size vehicles first, and then the priority is given to distributing vehicles from the rescue points that take a shorter travel time until the number of rescue vehicles in demand is satisfied. In this process, a penalty coefficient is used to make the scheduling work more flexible. The rescue professional organization can adjust the punishment coefficient according to factors such as the level of the tunnel collapse accident and the number of people trapped. A nondominated sorting genetic algorithm with elite strategy (NSGA-II) is proposed to solve the multi-objective model in the second stage. The NSGA-II algorithm has the characteristics of good solution distribution and fast convergence speed and can acquire serval high-quality solutions after one run time. Compared with other algorithms, the performance of the NSGA-II algorithm is significantly improved. In the process of the NSGA-II algorithm, the maximum number of iterations is set as $r_{\max }$ first, and then the initial population, $P_{r}(r=0)$, is randomly generated with the size of $n$. Secondly, the function values of each individual $P_{r}$ are calculated and sorted by non-dominance. Hence, the subpopulations of the t-generation population are obtained by using the selection, crossover, and mutation operations in genetic operators, denoted by $Q_{r}$. Thirdly, $P_{r}$ and $Q_{r}$ are merged as $P_{r} \cup Q_{r}$. According to the non-dominated relationship and individual crowding degree, the elite strategy is adopted to select $n$ individuals from the set $P_{r} \cup Q_{r}$ to form the parent population $P_{r+1}$. Finally, the new population is generated by genetic operators again, as mentioned before. Repeat the above steps until the maximum number of iterations, $r_{\max }$, is reached, and output the Pareto optimal solution set.

The steps of developing this non-dominated sorting genetic algorithm (NSGA-II) are explained in detail as follows:

Step 1: Coding design

In association with the multiple rescue points and various types of vehicles, the coding method in this paper is designed as a real weight type. The serial numbers corresponding to different rescue points and vehicle sizes are coded. Thus, the decoding operation is unnecessary, leading to the improvement of the solution's accuracy and speed. Due to this, the convergence of the algorithm is improved as it is convenient to add real genetic operators.

The initial chromosome population is $P_{r}(r=0)$. Each chromosome contains two substrings: $P_{t}^{i}=\left[P_{t}^{i 1}, P_{t}^{i 2}\right], i=1,2, \ldots, p o p$. These two substrings are generated as follows: $P_{r}^{i 1}=\operatorname{randint}(1,1,[0, u])+0.01 \times \operatorname{randint}(1,1,[0,100]), P_{r}^{i 2}=\operatorname{randint}\left(1,1,\left[0, u_{j}\right]\right) u_{j} . u$ and $u_{j}$ are the number of rescue points and their vehicles in each rescue point, respectively. $P_{r}^{i 1}$ is a randomly generated real number with two decimal places, and $P_{r}^{i 2}$ is a randomly generated positive integer. A set of decision variables as shown in Table 5 is taken as an example to explain further. 
Table 5. Coding operation example.

\begin{tabular}{cccc}
\hline Gene & Rescue Point & Vehicle Size & Scheduling Amount \\
\hline$[4.21,3]$ & 4 & 1 & 3 \\
{$[5.41,4]$} & 5 & 2 & 4 \\
$\ldots$ & 7 & $\ldots$ & 5 \\
\hline $7.82,5]$ & & 3 & \\
\hline
\end{tabular}

From Table 5, for gene [4.21, 3], the integer part of 4.21 indicates that the rescue point is $j=4$, and the fractional part is the probability determined by the roulette algorithm when choosing the vehicle size. For example, the rescue point has three sizes of vehicles (size 1, size 2, size 3), and the probability of each is $\frac{1}{3}$. For the fractional part, of which the value is 0.21 , it is in the probability interval $[0,0.33]$, leading to the vehicle size selected being size 3 . As for the number scheduled, the latter part helps to provide the answer. Gene [4.21,3] represents the scheduling plan; that is, distributing 3 vehicles which are of size 3 from the rescue point 4 . One gene depicts one scheduling plan.

Step 2: Fitness function

The objective function after normalization, as shown in Equation (24), is implemented here as the fitness function.

Step 3: The selection, crossover, and mutation of genetic operators

(1) Selecting operator: the fitness ratio method is used for the selection operation. After sorting the fitness value of each individual, the individuals have been selected. The higher the fitness value is, the higher the probability that the individual will be selected.

$$
p_{i}^{b s}=\frac{f_{i}}{\sum_{i=1}^{N} f_{i}}
$$

where $p_{i}^{b s}$ is the probability of an individual $i$ being selected, $N$ represents the population size, and $f_{i}$ represents the fitness value of the individual $i$.

(2) Operator crossover: the vector linear combination method is used in the crossover operation to generate the offspring generations:

$$
\begin{aligned}
& E_{a^{\prime}}=\varepsilon e_{a}+(1-\varepsilon) e_{b} \\
& E_{b^{\prime}}=\varepsilon e_{b}+(1-\varepsilon) e_{a}
\end{aligned}
$$

where $e_{a}$ and $e_{b}$, respectively, represent the two individuals to be crossed, $0<\varepsilon<1$.

(3) Mutation operator: The method of reverse mutation is used here. Two positions were randomly selected from the chromosome first, and then the substring between them was reversed.

Step 4: Termination condition

The algorithm stops when the running algebra reaches the set maximum cap $n$. The solution process is shown in Figure 1. 


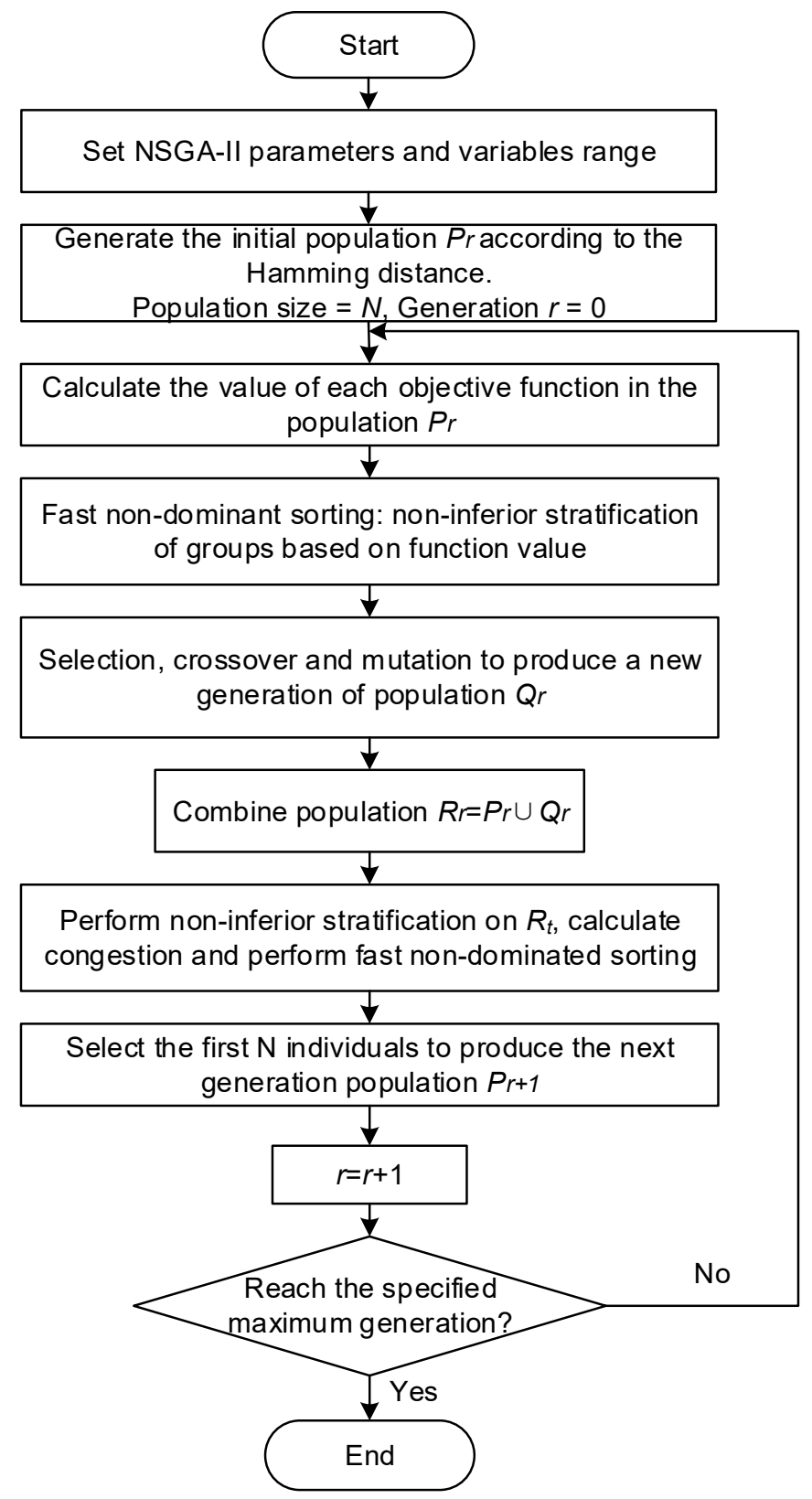

Figure 1. Solution flow chart.

\section{Case Study}

In this section, we first apply the proposed model to a real-size example to verify its practicability and adaptability. At the same time, we conduct the same decision-making process on the example without the difference of vehicle sizes to observe the improvement effect of the proposed model. Then, we make a comparison of different coding methods for the NSGA-II algorithm used in this paper.

\subsection{Example Description}

A tunnel under construction is taken as a real example in this paper. There are 23 nodes and 27 road sections in the corresponding topology transportation network, as shown in Figure 2. For reducing the complexity of the calculation, the nearby hospital and fire station (distance $<1 \mathrm{~km}$ ) are treated as one rescue point. The tunnel where the collapse occurred is located near node 23 . There are road restrictions on road sections 11 and 20 so that large 
vehicles cannot pass through. The design speed of all sections is set as $v_{a}^{d m}=120 \mathrm{~km} / \mathrm{h}$. The total amount of supplies required for rescue is $b=4000 \mathrm{~m}^{3}$.

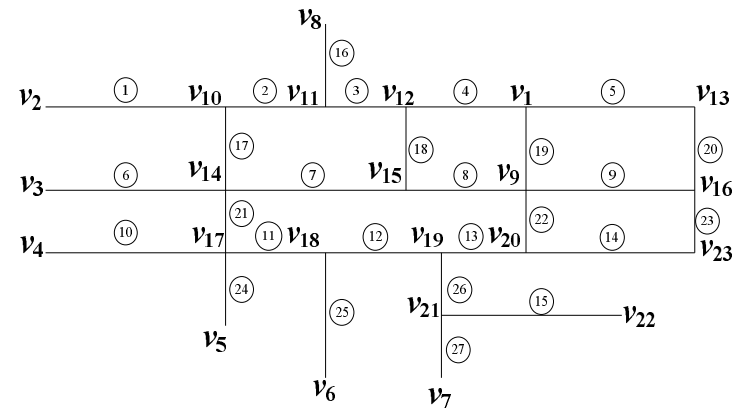

Figure 2. Transportation network.

The original number of vehicles available for scheduling at the rescue points $v_{1}-v_{9}$ and other parameter settings are shown in Tables 6 and 7.

Table 6. Number of vehicles available at rescue points.

\begin{tabular}{cccc}
\hline Rescue Point & Small & $\begin{array}{c}\text { Quantity } \\
\text { Medium }\end{array}$ & Large \\
\hline 1 & 14 & 12 & 12 \\
2 & 12 & 13 & 13 \\
3 & 15 & 12 & 16 \\
4 & 13 & 12 & 14 \\
5 & 13 & 12 & 14 \\
6 & 15 & 13 & 12 \\
7 & 13 & 11 & 14 \\
8 & 14 & 15 & 16 \\
9 & 12 & 13 & 12 \\
\hline
\end{tabular}

Table 7. Other parameters.

\begin{tabular}{|c|c|c|c|}
\hline Size & Small & Medium & Large \\
\hline$q^{m}\left(\mathrm{~m}^{3}\right)$ & 8 & 25 & 57 \\
\hline$c_{t}($ yuan $/ \mathrm{m})$ & 0.1 & 0.2 & 0.3 \\
\hline first stage $d_{m}^{f}$ & 17 & 10 & 12 \\
\hline sec ond stage $d_{m}^{s}$ & 14 & 10 & 20 \\
\hline
\end{tabular}

\subsection{Vehicle Scheduling Comparison}

According to the Equations (1)-(10), the travel times of the three sizes of vehicles in different road sections with special conditions were calculated. The results of sections 1-10 are listed in Table 8. 
Table 8. Section information and vehicle travel time.

\begin{tabular}{|c|c|c|c|c|c|c|c|c|c|c|}
\hline $\begin{array}{l}\text { Road } \\
\text { Section }\end{array}$ & $\begin{array}{l}\text { Section } \\
\text { Length }\end{array}$ & $\begin{array}{l}\text { The Radius of the } \\
\text { Horizontal Curve }\end{array}$ & Slope & $\begin{array}{l}\text { Slope } \\
\text { Length }\end{array}$ & $\begin{array}{l}\text { Traffic } \\
\text { Flow }\end{array}$ & Traffic Flow Composition & $P C I$ & Small & $\begin{array}{l}\text { Travel Time/h } \\
\text { Medium }\end{array}$ & Large \\
\hline 1 & 0.825 & 400 & 2 & 0.6 & 567.84 & 0.2 & 78 & 0.45 & 0.68 & 0.73 \\
\hline 2 & 0.15 & 650 & 1.4 & 0.15 & 567.84 & 0 & 78 & 0.21 & 0.35 & 0.4 \\
\hline 3 & 0.15 & 780 & 1.4 & 0.15 & 663.54 & 0 & 78 & 0.22 & 0.41 & 0.53 \\
\hline 4 & 0.125 & 680 & 1.2 & 0.1 & 585.45 & 0 & 53 & 0.19 & 0.23 & 0.37 \\
\hline 5 & 0.075 & 850 & 0 & 0 & 585.48 & 0 & 45 & 0.04 & 0.14 & 0.23 \\
\hline 6 & 0.225 & 950 & 1.4 & 0.12 & 781.4 & 0 & 37 & 0.36 & 0.45 & 0.46 \\
\hline 7 & 0.5 & 550 & 0.2 & 0.5 & 781.4 & 0.2 & 54 & 0.3 & 0.64 & 0.78 \\
\hline 8 & 0.075 & 780 & 1.2 & 0.075 & 663.54 & 0 & 75 & 0.05 & 0.18 & 0,3 \\
\hline 9 & 0.1 & 780 & 0 & 0 & 663.54 & 0 & 76 & 0.07 & 0.25 & 0.32 \\
\hline 10 & 0.25 & 580 & 2 & 0.25 & 663.54 & 0.1 & 36 & 0.36 & 0.43 & 0.56 \\
\hline
\end{tabular}

We established the solution algorithm using Matlab R2014b (MathWorks, MA, USA) to solve the proposed model in this paper. The initial NSGA-II control parameters were set as: population size $N=50$, the number of iterations $n=300$, the crossover probability $p^{c r}=0.9$, and the mutation probability $p^{m u}=0.09$. The approximate Pareto front of the second stage is shown in Figure 3. Five groups of non-inferior solutions were selected in the second stage's solution set to calculate the three corresponding optimal objective function values, as shown in Table 9.

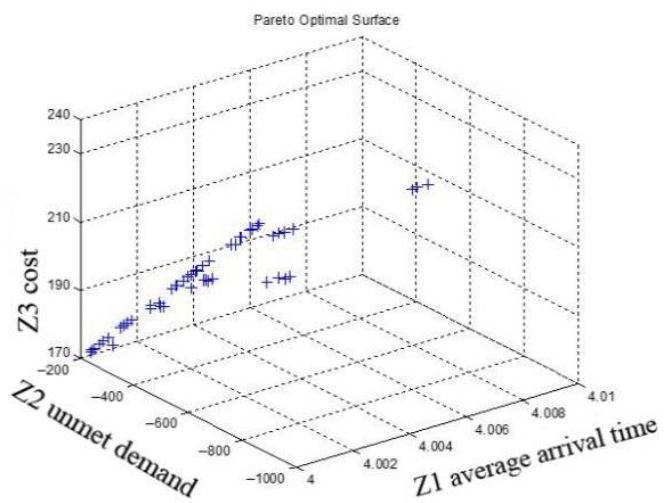

Figure 3. Pareto frontier map.

Table 9. Five groups of Pareto optimal solutions of the second stage.

\begin{tabular}{cccc}
\hline \multirow{2}{*}{ Plan } & Time & $\begin{array}{c}\text { Objective Function Value } \\
\text { Unmet Demand }\end{array}$ & Cost \\
\hline 1 & 4.003 & -221.56 & 197.52 \\
2 & 4.006 & -267.93 & 190.78 \\
3 & 4.008 & -241.57 & 190.65 \\
4 & 4.007 & -221.78 & 193.67 \\
5 & 4.005 & -278.89 & 199.85
\end{tabular}

Note: It means distributing and transporting redundant supplies when the unmet demand in the above table is negative.

The decision-makers can select the scheduling plan from the non-inferior solution according to the actual situation. Plan 1 was chosen as the optimal scheduling plan according to the vehicles' shortest arrival time, as shown in Table 10. 
Table 10. Scheduling plan for small, medium, and large vehicles at each rescue point.

\begin{tabular}{ccc}
\hline Size & The First-Stage Scheduling Plan & $\begin{array}{c}\text { The-Second Stage } \\
\text { Scheduling Plan }\end{array}$ \\
\hline Small & $\{(1,4)(7,3)(2,5)(9,4)(3,6)(4,3)(8,4)\}$ & $\{(3,2)(2,3)(7,3)(4,3)(9,1)\}$ \\
Medium & $\{(1,5)(6,2)(3,2)(7,5)(9,3)(4,5)(8,3)\}$ & $\{(1,3)(7,1)(3,2)(9,1)(8,2)\}$ \\
Large & $\{(1,3)(2,3)(3,3)(7,2)(9,3)(6,3)\}$ & $\{(1,3)(9,2)(3,5)(2,3)(7,2)(8,2)\}$ \\
\hline
\end{tabular}

Note: $\{(1,4)\}$ means to distribute 4 vehicles from rescue point 1 in the above table.

We removed the difference between the vehicle sizes in the model to investigate the improved effect in this section. For example, in Section 4.1, the objective function without the multi-size method is shown in Table 11.

Table 11. Five groups of Pareto optimal solutions of the second stage without the multi-size method.

\begin{tabular}{cccc}
\hline Plan & Time & $\begin{array}{c}\text { Objective Function Value } \\
\text { Unmet Demand }\end{array}$ & Cost \\
\hline 1 & 3.996 & -300.71 & 203.54 \\
2 & 3.904 & -289.56 & 201.37 \\
3 & 3.900 & -341.74 & 210.32 \\
4 & 3.901 & -321.44 & 204.85 \\
5 & 3.899 & -365.83 & 207.41 \\
\hline
\end{tabular}

From Table 11, it can be seen that without the difference of the vehicles, the time objective is decreased, because the rescue vehicles near the affected node will be scheduled instead of those in the farther rescue points without the limitation of the vehicles. Although the time is shortened, this leads to a negative change of the unmet demand objective, since some of the vehicles transporting supplies will be influenced by the actual road conditions. Moreover, the distant rescue vehicles are dismissed by the shortest arrival time limitation. The cost objective function has not changed much.

\subsection{Comparison of Algorithm Efficiency}

In this section, we first explain the improvement of the NSGA-II algorithm applied in this paper and then compare the efficiency of the NSGA-II algorithm used in this study, the multi-objective gray wolf algorithm, and the general genetic algorithm based on the real number coding method. The proposed model in this paper involves multiple rescue points and multiple vehicle sizes, which show the complexity of the problem. A genetic algorithm is a common algorithm for solving such problems. This paper choses the NSGA-II algorithm to solve the problem instead of the general genetic algorithm (GA). This algorithm considers all the frontiers, which reflects the diversity of the solution, and therefore the solutions can effectively prevent falling into the local optimum. In addition, the use of elite strategies can speed up convergence and remove inferior solutions faster. These two operations interact to find a suitable convergence speed as soon as possible, reduce the calculation time, and avoid falling into the local optimum. Binary coding is often used when dealing with optimization problems. Although, their structures may be very different when representing adjacent integers, leading to the low efficiency of genetic operators. Moreover, due to the long length of the general variable, the operations of constantly coding and decoding in the process of calculation are unavoidable, and then the calculation time is prolonged. Therefore, we chose the real number coding method in this study.

In this paper, we applied real number coding to the NSGA algorithm and compared it with the multi-objective grey wolf algorithm (MOGWO) and the traditional genetic algorithm (GA) in terms of objective function value, the number of iterations, and running time. During the experiments, the coding, population size, and the number of iterations were set consistently for the three algorithms to ensure fairness and objectivity. For the multi-objective grey wolf algorithm [32], the real number was coded to represent the 
position of each wolf. Multi-size vehicles and multiple rescue points were considered in this study, as mentioned above, which led to the increase of dimensionality and the number of individuals. Therefore, this brings about the geometric growth increase of complexity in the general genetic algorithm. The results of the comparison are shown in Table 12.

Table 12. Algorithm efficiency comparison.

\begin{tabular}{cccc}
\hline Algorithm & Objective Value & $\begin{array}{c}\text { Iteration Frequency } \\
\text { (Times) }\end{array}$ & Run Time (s) \\
\hline $\begin{array}{c}\text { General genetic } \\
\text { algorithm }\end{array}$ & -28.53 & 215 & 97 \\
$\begin{array}{c}\text { Multi-objective gray } \\
\text { wolf algorithm }\end{array}$ & -31.05 & 56 & 59 \\
NSGA-II algorithm & -31.53 & 45 & 46 \\
\hline
\end{tabular}

It can be seen that the objective value of NSGA-II was smaller than both the multiobjective grey wolf algorithm and the traditional genetic algorithm. It was $9 \%$ lower than that of the genetic algorithm and is similar to the competitive multi-objective grey wolf algorithm, reflecting the superiority of the algorithm used in this paper. The number of iterations was reduced by $79 \%$ and $24 \%$, respectively, and the speed of reaching the optimal solution was increased by $53 \%$ and $22 \%$. In summary, the NSGA-II method designed for this paper can accelerate the convergence of the objective function values and outperform the multi-objective grey wolf algorithm and the traditional genetic algorithm.

\section{Conclusions}

This paper proposed a theory to optimize the scheduling plan of the rescue vehicles after a tunnel collapse in view of the different vehicles' sizes and the priority of the objectives in different stages. The rescue vehicles were divided into three sizes, including small, medium, and large, and then the speed corrections on the road with special conditions were performed for each size of the vehicle. A multi-objective model determining the scheduling plan of three sizes of vehicles from the rescue points was developed. In the first stage, the objective of minimizing the maximum completion time of scheduling ensures the priority of timely assistance to the trapped people. In the second stage, with the main aim of reconstruction, the scheduling time, unmet demand, and the total scheduling cost are comprehensively considered. A non-dominated sorting genetic algorithm with elite strategy (NSGA-II) with the method of real number coding was proposed to solve the model, and a real case study was provided to evaluate the proposed model's effectiveness. A comparison between multi-size and simple-size based on the case study was conducted, and the result showed that compared with the simple-size, the multi-size method comprehensively ensured the three objectives. Therefore, the efficiency and level of the rescue operations can be significantly enhanced, and the human suffering and the economic loss can be mitigated. Moreover, a comparison was carried out to investigate the efficiency of the three algorithms. The numerical experimental results showed that compared with the general genetic algorithm and the multi-objective gray wolf algorithm, the convergence speed of real coding was $53 \%$ and $22 \%$ faster, respectively. Hence, verifying the optimization performance of the algorithm in this paper.

In future research, the work in this paper can be further studied in the following aspects. First, the speed correction can be adjusted to acquire a more accurate value. Secondly, given the complexity of solving large-scale networks, the efficiency of algorithms applied in this work could be evaluated in terms of more quality indicators based on the previous research [33]. Thirdly, as well as tunnel collapse, it is expected to extend the research idea in this paper to other disasters such as earthquakes and hurricanes. Finally, it will be interesting to integrate the proposed model into the optimal emergency management process to fulfill the emergency system. 


\begin{abstract}
Author Contributions: H.C.: oversight and leadership responsibility for the research activity planning and execution; Y.Y. developed the model and analyzed the data; L.L. performed the research and wrote the paper; M.Z. revised the manuscript. All authors contributed to revising and refining the ideas, carrying out additional analyses, and finalizing this paper. All authors have read and agreed to the published version of the manuscript.
\end{abstract}

Funding: This research was funded by the National Natural Science Foundation of China, grant number 52172304, the Hebei Province Technology Innovation Guidance Program, grant number 19970808D. and the High-level Talent Funding Project of Hebei Provincial Department of Human Resources and Social Security, grant number A201905003. And The APC was funded by Hongjun Cui.

Conflicts of Interest: The authors declare no conflict of interest.

\title{
References
}

1. Cheng, G. Research on Speed Limiting Method on Expressway; Harbin Institute of Technology: Harbin, China, 2007. (In Chinese)

2. Barbarosoğlu, G.; Özdamar, L.; Çevik, A. An interactive approach for hierarchical analysis of helicopter logistics in disaster relief operations. Eur. J. Oper. Res. 2002, 140, 118-133. [CrossRef]

3. De Angelis, V.; Mecoli, M.; Nikoi, C.; Storchi, G. Multiperiod integrated routing and scheduling of World Food Programme cargo planes in Angola. Comput. Oper. Res. 2007, 34, 1601-1615. [CrossRef]

4. Grass, E.; Fischer, K. Two-stage stochastic programming in disaster management: A literature survey. Surv. Oper. Res. Manag. Sci. 2016, 21, 85-100. [CrossRef]

5. Erbeyoğlu, G.; Bilge, Ü. A robust disaster preparedness model for effective and fair disaster response. Eur. J. Oper. Res. 2020, 280, 479-494. [CrossRef]

6. Alem, D.; Clark, A.; Moreno, A. Stochastic network models for logistics planning in disaster relief. Eur. J. Oper. Res. 2016, 255, 187-206. [CrossRef]

7. Bozorgi-Amiri, A.; Khorsi, M. A dynamic multi-objective location-routing model for relief logistic planning under uncertainty on demand, travel time, and cost parameters. Int. J. Adv. Manuf. Technol. 2016, 85, 1633-1648. [CrossRef]

8. Cavdur, F.; Kose-Kucuk, M.; Sebatli, A. Allocation of temporary disaster response facilities under demand uncertainty: An earthquake case study. Int. J. Disaster Risk Reduct. 2016, 19, 159-166. [CrossRef]

9. Condeixa, L.D.; Leiras, A.; Oliveira, F.; de Brito, I. Disaster relief supply pre-positioning optimization: A risk analysis via shortage mitigation. Int. J. Disaster Risk Reduct. 2017, 25, 238-247. [CrossRef]

10. Fereiduni, M.; Shahanaghi, K. A robust optimization model for distribution and evacuation in the disaster response phase. J. Ind. Eng. Int. 2017, 13, 117-141. [CrossRef]

11. Jin, S.; Jeong, S.; Kim, J.; Kim, K. A logistics model for the transport of disaster victims with various injuries and survival probabilities. Ann. Oper. Res. 2015, 230, 17-33. [CrossRef]

12. Pradhananga, R.; Mutlu, F.; Pokharel, S.; Holguín-Veras, J.; Seth, D. An integrated resource allocation and distribution model for pre-disaster planning. Comput. Ind. Eng. 2016, 91, 229-238. [CrossRef]

13. Vitoriano, B.; Ortuño, M.T.; Tirado, G.; Montero, J. A multi-criteria optimization model for humanitarian aid distribution. J. Glob. Optim. 2011, 51, 189-208. [CrossRef]

14. Wassenhove, L.N.V.; Martinez, A.J.P. Using OR to adapt supply chain management best practices to humanitarian logistics. Int. Trans. Oper. Res. 2012, 19, 307-322. [CrossRef]

15. Barzinpour, F.; Esmaeili, V. A multi-objective relief chain location distribution model for urban disaster management. Int. J. Adv. Manuf. Technol. 2014, 70, 1291-1302. [CrossRef]

16. Dalal, J.; Üster, H. Robust Emergency Relief Supply Planning for Foreseen Disasters Under Evacuation-Side Uncertainty. Transp. Sci. 2021, 55, 791-813. [CrossRef]

17. Doodman, M.; Shokr, I.; Bozorgi-Amiri, A.; Jolai, F. Pre-positioning and dynamic operations planning in pre- and post-disaster phases with lateral transhipment under uncertainty and disruption. J. Ind. Eng. Int. 2019, 15, 53-68. [CrossRef]

18. Elçi, Ö.; Noyan, N. A chance-constrained two-stage stochastic programming model for humanitarian relief network design. Transp. Res. Part B Methodol. 2018, 108, 55-83. [CrossRef]

19. Paul, J.A.; MacDonald, L. Location and capacity allocations decisions to mitigate the impacts of unexpected disasters. Eur. J. Oper. Res. 2016, 251, 252-263. [CrossRef]

20. Di Pasquale, V.; Fruggiero, F.; Iannone, R. A numerical approach for inventory pre-positioning in emergency management. J. Humanit. Logist. Supply Chain Manag. 2020, 10, 205-230. [CrossRef]

21. Mohamadi, A.; Yaghoubi, S. A bi-objective stochastic model for emergency medical services network design with backup services for disasters under disruptions: An earthquake case study. Int. J. Disaster Risk Reduct. 2017, 23, 204-217. [CrossRef]

22. Tofighi, S.; Torabi, S.A.; Mansouri, S.A. Humanitarian logistics network design under mixed uncertainty. Eur. J. Oper. Res. 2016, 250, 239-250. [CrossRef]

23. Ali Torabi, S.; Shokr, I.; Tofighi, S.; Heydari, J. Integrated relief pre-positioning and procurement planning in humanitarian supply chains. Transp. Res. Part E Logist. Transp. Rev. 2018, 113, 123-146. [CrossRef] 
24. Huang, K.; Jiang, Y.; Yuan, Y.; Zhao, L. Modeling multiple humanitarian objectives in emergency response to large-scale disasters. Transp. Res. Part E Logist. Transp. Rev. 2015, 75, 1-17. [CrossRef]

25. Noham, R.; Tzur, M. Designing humanitarian supply chains by incorporating actual post-disaster decisions. Eur. J. Oper. Res. 2018, 265, 1064-1077. [CrossRef]

26. Berkoune, D.; Renaud, J.; Rekik, M.; Ruiz, A. Transportation in disaster response operations. Socio-Econ. Plan. Sci. 2012, 46, 23-32. [CrossRef]

27. Norouzi, N.; Sadegh-Amalnick, M.; Tavakkoli-Moghaddam, R. Modified particle swarm optimization in a time-dependent vehicle routing problem: Minimizing fuel consumption. Optim. Lett. 2017, 11, 121-134. [CrossRef]

28. Hongtao, S.; Yucai, D.; Lianghai, Y.; Dongyun, Z.; Hong, J.; Erchang, M.; Weidong, L. Study on the Route Optimization of Military Logistics Distribution in Wartime Based on the Ant Colony Algorithm. Comput. Inf. Sci. 2010, 3, 139. [CrossRef]

29. Mohammadi, R.; Ghomi, S.M.T.F.; Jolai, F. Prepositioning emergency earthquake response supplies: A new multi-objective particle swarm optimization algorithm. Appl. Math. Model. 2016, 40, 5183-5199. [CrossRef]

30. Dalal, J.; Üster, H. Combining Worst Case and Average Case Considerations in an Integrated Emergency Response Network Design Problem. Transp. Sci. 2018, 52, 171-188. [CrossRef]

31. Jin, B.; Bi, L. Multi-objective workshop scheduling based on genetic algorithm. Modul. Mach. Tool Autom. Manuf. Technol. 2019, 4, 157-160.

32. Mirjalili, S.; Mirjalili, S.M.; Lewis, A. Grey Wolf Optimizer. Adv. Eng. Softw. 2014, 69, 46-61. [CrossRef]

33. Li, M.; Yao, X. Quality Evaluation of Solution Sets in Multiobjective Optimisation: A Survey. ACM Comput. Surv. 2019, 52, 1-38. [CrossRef] 